\title{
A Case of Xanthogranulomatous Cholecystitis Preoperatively Diagnosed with Contrast-enhanced Ultrasonography
}

\author{
Junji Ueda ${ }^{1,2}$, Hiroshi Yoshida ${ }^{1,2}$, Yasuo Arima ${ }^{1,2}$, Yasuhiro Mamada ${ }^{1,2}$, \\ Nobuhiko Taniai ${ }^{1,2}$, Sho Mineta ${ }^{1,2}$, Masato Yoshioka ${ }^{1,2}$, Youichi Kawano ${ }^{1,2}$, \\ Zenya Naito ${ }^{3}$ and Eiji Uchida ${ }^{1,2}$ \\ ${ }^{1}$ Surgery for Organ Function and Biological Regulation, Graduate School of Medicine, Nippon Medical School \\ ${ }^{2}$ Department of Surgery, Nippon Medical School \\ ${ }^{3}$ Department of Pathology, Nippon Medical School
}

\begin{abstract}
We report a case of xanthogranulomatous cholecystitis (XGC) that was diagnosed preoperatively by means of ultrasonography (US) with the contrast-enhancement agent Sonazoid after a false-positive result had been obtained with fluorine-18 fluorodeoxyglucose positron emission tomography (FDG-PET). A 69-year-old woman was admitted because of right upper quadrant pain. Blood tests revealed a serum CA19-9 level of 749.8 IU/L. Computed tomography (CT), US, and magnetic resonance imaging of the abdomen showed abnormal thickening of the gallbladder wall but no stones. The border between the gallbladder and the liver was unclear. FDG-PET revealed a lesion with increased uptake of tracer in the gallbladder wall. The thickness of the lesion was similar to that on CT. We suspected gallbladder carcinoma with hepatic invasion. To confirm the tentative diagnosis, we performed US with the contrast-enhancement agent Sonazoid. The gallbladder wall was homogeneously enhanced in the early vascular phase and remained enhanced for 90 seconds. Enhancement of the gallbladder wall was smooth and regular. The border between the gallbladder and liver was clear and smooth. On the basis of these examinations, we diagnosed chronic cholecystitis (XGC suspected), not gallbladder carcinoma. At surgery, the gallbladder wall was observed to be extremely thick because of severe inflammation, and cholecystectomy was performed. XGC was diagnosed on intraoperative pathological examination. Histopathological examination showed XGC, severe proliferative fibrosis with formation of multiple yellow-brown intramural nodules, and foamy histiocytes without malignant cells. In conclusion, the present case of XGC was diagnosed preoperatively with contrast-enhanced US after a false-positive result had been obtained with FDG-PET. Contrast-enhanced US can thus play important roles in diagnosing gallbladder disease and selecting treatment.

(J Nippon Med Sch 2011; 78: 194-198)
\end{abstract}

Key words: xanthogranulomatous cholecystitis, contrast-enhanced ultrasonography, positron emission tomography

Correspondence to Junji Ueda, Department of Surgery, Nippon Medical School, 1-1-5 Sendagi, Bunkyo-ku, Tokyo 113-8603, Japan

E-mail: junji0821@nms.ac.jp

Journal Website (http://www.nms.ac.jp/jnms/) 


\section{Introduction}

Xanthogranulomatous cholecystitis (XGC) is a rare inflammatory lesion of the gallbladder, characterized by marked proliferative fibrosis, macrophage infiltration, and foam cells involving the gallbladder wall $^{1}$. It is often difficult to distinguish XGC from gallbladder carcinoma by means of conventional imaging techniques, such as ultrasonography (US), computed tomography (CT), and magnetic resonance imaging ${ }^{2-7}$. Some patients with XGC have undergone excessive surgical resection after XGC was misdiagnosed preoperatively as advanced gallbladder carcinoma ${ }^{8,9}$.

Several recent studies have demonstrated that fluorine-18 fluorodeoxyglucose positron emission

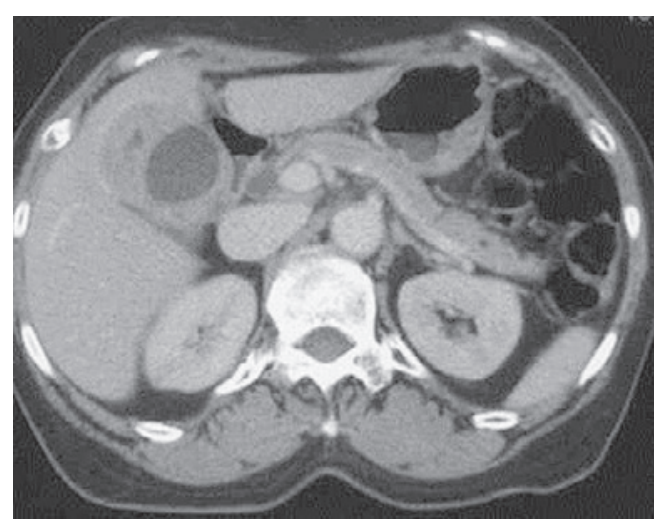

Fig. 1 CT showed an abnormally thickened gallbladder wall without stones. Invasion of the liver bed was suspected.

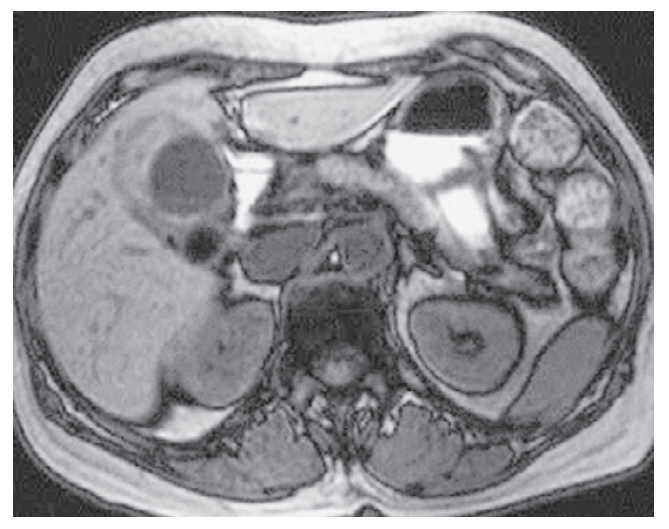

Fig. 2 Magnetic resonance imaging (T1-weighted) showed an abnormally thickened gallbladder wall without gallstones. Invasion of the liver bed was suspected. tomography (FDG-PET) is useful for distinguishing between benign and malignant lesions in the gallbladder $^{10-14}$. However, FDG-PET is not specific for malignant lesions, and tracer can accumulate in inflammatory lesions with increased glucose metabolism ${ }^{15}$. Contrast-enhanced US is reportedly useful for diagnosing gallbladder lesions, particularly for distinguishing malignant and benign lesions ${ }^{16}$. We report a case of XGC that was diagnosed preoperatively by means of US with the contrastenhancement agent Sonazoid (Daiichi Sankyo Co., Tokyo, Japan), after a false-positive result had been obtained with FDG-PET.

\section{Case Report}

A 69-year-old woman was admitted because of right upper quadrant pain. An abdominal CT scan revealed thickening of the gallbladder wall. The past history included thymoma, which was resected 5 years earlier, and pure red-cell aplasia, which was not treated medically. Blood tests revealed a serum CA19-9 level of $749.8 \mathrm{IU} / \mathrm{L}$ (normal, <37 IU/L). Magnetic resonance, US, and CT of the abdomen showed abnormal thickening of the gallbladder wall but no gallstones. The border between the gallbladder and liver was unclear (Fig. 1, 2). FDGPET revealed a lesion with increased uptake of tracer in the gallbladder wall. The thickness of the lesion was similar to that on CT (Fig. 3). Gallbladder carcinoma with hepatic invasion was suspected. To confirm the diagnosis, we performed US with the

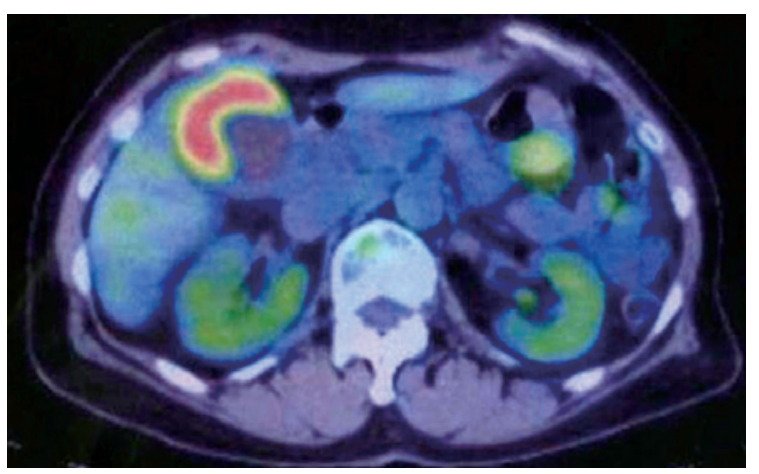

Fig. 3 FDG-PET revealed a lesion with increased uptake of tracer in the gallbladder wall. The thickness of the lesion was similar to that on CT. 

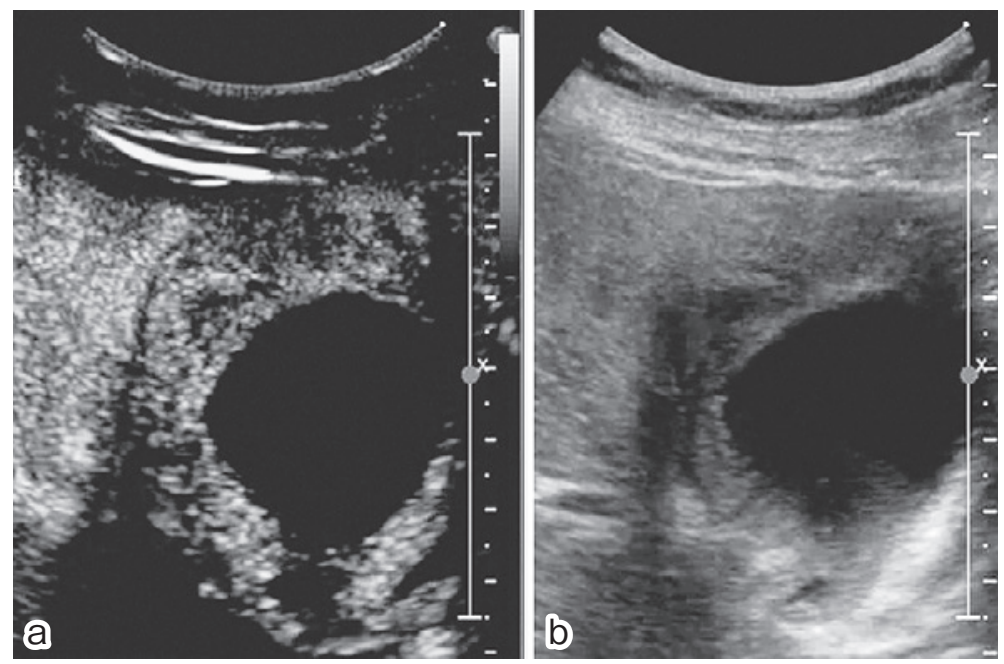

Fig. 4 US enhanced with the contrast agent Sonazoid showed that the gallbladder wall was homogeneously enhanced in the early vascular phase and remained enhanced for 90 seconds. Enhancement of the gallbladder wall was smooth and regular. The border between the gallbladder and liver was clear and smooth.

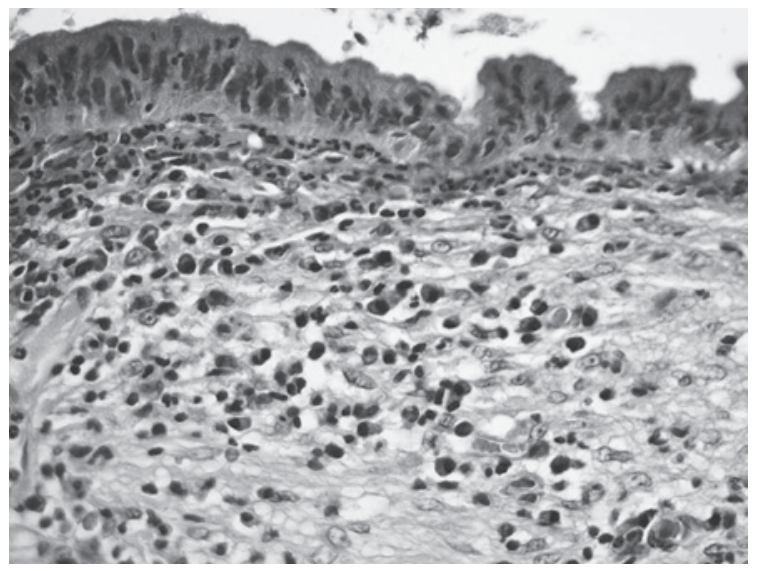

Fig. 5 Pathological examination revealed XGC, severe proliferative fibrosis with formation of multiple yellow-brown intramural nodules, and foamy histiocytes without malignant cells.

contrast agent Sonazoid. The gallbladder wall was homogeneously enhanced in the early vascular phase and remained enhanced for 90 seconds. Enhancement of the gallbladder wall was smooth and regular. The border between the gallbladder and liver was clear and smooth (Fig. 4). On the basis of these results, we diagnosed chronic cholecystitis (XGC suspected), not gallbladder carcinoma.

At surgery, the gallbladder wall was observed to be extremely thick because of severe inflammation, and cholecystectomy was performed. XGC was diagnosed on intraoperative pathological examination. Histopathological examination revealed $\mathrm{XGC}$, severe proliferative fibrosis with formation of multiple yellow-brown intramural nodules, and foamy histiocytes without malignant cells (Fig. 5). The postoperative course was uneventful, and the patient was discharged on postoperative day 14 .

\section{Discussion}

XGC was first reported and named by McCoy and colleagues ${ }^{17}$. It accounts for only $0.7 \%$ to $13.2 \%$ of all inflammatory diseases of the gallbladder and occurs predominantly in middle-aged and elderly persons ${ }^{18,19}$. The low incidence of XGC sometimes leads to misdiagnosis ${ }^{20}$.

$\mathrm{XGC}$ is characterized by a destructive inflammatory process of the gallbladder, associated with deposition of bile pigments and cholesterol in the gallbladder wall. Macroscopically, lesions vary from a small limited focus within a yellow-brown nodule in the gallbladder wall to diffuse involvement of the entire gallbladder with extension to surrounding structures ${ }^{21}$. Gallbladder stones were present in $85 \%$ to $100 \%$ of patients in different series, with obstruction of the cystic duct in $80 \%$ of 
cases $^{1,18}$. The pathogenesis of XGC is uncertain, but the current opinion favors a combination of acute inflammation of the gallbladder and outflow obstruction due to gallbladder stones ${ }^{18,22}$. Bile enters the stroma of the gallbladder wall through ruptured Rokitansky-Aschoff sinuses or mucosal ulcerations due to gallbladder stones, acute inflammation, or both. Extravasated bile in the stroma causes intense inflammatory reactions with accumulation of histiocytes, which engulf insoluble cholesterol and other bile lipids to form large, round xanthoma cells. Microabscesses form in the gallbladder wall, eventually resulting in xanthogranulomata. Finally, a fibrous reaction and scarring result from healing of the inflamed tissue ${ }^{23}$. Both intramural poorly echogenic nodulated zones on US and intramural hypoattenuated nodules on CT are specific for XGC. Despite these distinctions, it is difficult to distinguish XGC from gallbladder carcinoma ${ }^{8}$.

Several recent studies have shown that FDG-PET is useful for differentiating between benign and malignant lesions in the gallbladder. The sensitivity and specificity of FDG-PET for the diagnosis of gallbladder carcinoma range from $75 \%$ to $100 \%$ and $75 \%$ to $89 \%$, respectively ${ }^{10-14}$. However, FDG-PET is not specific for malignant lesions, and tracer can accumulate in inflammatory lesions with increased glucose metabolism. Indeed, false-positive results have been obtained with benign lesions, including chronic cholecystitis, tuberculosis, and adenomyomatosis of the gallbladder ${ }^{13,24,25}$. In our patient, FDG-PET/CT scans showed increased activity in the thickened wall of the gallbladder which was associated with an inflammatory, not malignant, lesion. In the near future, FDG-PET may used more frequently to diagnose gallbladder carcinoma. We should consider the risk of misdiagnosing inflammatory lesions as carcinoma, because inflammatory diseases occur frequently in the gallbladder ${ }^{15}$.

Contrast-enhanced US has been reported to be useful for diagnosing gallbladder lesions, particularly for differentiating malignant and benign lesions ${ }^{16}$. Contrast-enhanced US provides the advantages of real-time, repeatable, multiplanar imaging without compromising patient safety or exposing patients to radiation $^{26}$. When the tumor is more mass-like or the gallbladder wall is diffusely thickened, hyperenhancement followed by rapid washout of contrast agent within 35 seconds after administration is highly suggestive of gallbladder malignancy ${ }^{16}$. Disruption of gallbladder wall integrity, a feature not seen in benign gallbladder diseases, has been demonstrated in up to $85 \%$ of gallbladder carcinomas $^{16}$. Improved mural visualization after contrast-agent administration and the malignant feature of late-phase hypovascularity relative to the hepatic parenchyma might help to sharply delineate the tumor ${ }^{26}$. Hirooka et al. have reported that the 3layer structure of the gallbladder wall is intact on contrast-enhanced US in patients with cholecystitis. They suggested this finding will prove important for differentiating carcinoma from cholecystitis ${ }^{27}$. Numata et al. have reported that the observation of tumor vessels is useful for differentiating gallbladder carcinoma from adenomas, inflammatory polyps, and cholesterol polyps on contrast-enhanced harmonic $\mathrm{US}^{28}$. The arterial branches supplying a gallbladder carcinoma tend to show irregularly tortuous extension. Benign gallbladder lesions are also hypervascular and contain arteries of almost normal caliber which taper normally and subdivide normally into small vessels ${ }^{28}$. We performed contrast-enhanced US with Sonazoid. The gallbladder wall was homogeneously enhanced in the early vascular phase and remained enhanced for 90 seconds. The enhancement of the gallbladder wall was smooth and regular, no mucosal irregularity was detected, and the border with the liver was clear and smooth. The third layer of the gallbladder wall was intact. The arterial branches supplying this lesion did not show irregularly tortuous extension. These findings led to a diagnosis of chronic cholecystitis, (XGC suspected), not gallbladder carcinoma, and cholecystectomy was performed. The diagnosis on intraoperative pathological examination was also $\mathrm{XGC}$.

In conclusion, we have reported a case of XGC diagnosed preoperatively with contrast-enhanced US after a false-positive result had been obtained with FDG-PET. Contrast-enhanced US can thus play an important role in diagnosing gallbladder disease and 
selecting treatment.

\section{References}

1. Kwon AH, Matsui Y, Uemura Y: Surgical procedures and histopathologic findings for patients with xanthogranulomatous cholecystitis. J Am Coll Surg 2004; 199: 204-210.

2. Parra JA, Acinas O, Bueno J, Guezmes A, Fernandez MA, Farinas MC: Xanthogranulomatous cholecystitis: clinical, sonographic, and CT findings in 26 patients. AJR Am J Roentgenol 2000; 174: 979983.

3. Chun KA, Ha HK, Yu ES, et al.: Xanthogranulomatous cholecystitis: CT features with emphasis on differentiation from gallbladder carcinoma. Radiology 1997; 203: 93-97.

4. Kim PN, Lee SH, Gong GY, et al.: Xanthogranulomatous cholecystitis: radiologic findings with histologic correlation that focuses on intramural nodules. AJR Am J Roentgenol 1999; 172: 949-953.

5. Ros PR, Goodman ZD: Xanthogranulomatous cholecystitis versus gallbladder carcinoma. Radiology 1997; 203: 10-12.

6. Hatakenaka M, Adachi T, Matsuyama A, Mori M, Yoshikawa Y: Xanthogranulomatous cholecystitis: importance of chemical-shift gradient-echo MR imaging. Eur Radiol 2003; 13: 2233-2235.

7. Shuto R, Kiyosue H, Komatsu E, et al: CT and MR imaging findings of xanthogranulomatous cholecystitis: correlation with pathologic findings. Eur Radiol 2004; 14: 440-446.

8. Spinelli A, Schumacher G, Pascher A, et al.: Extended surgical resection for xanthogranulomatous cholecystitis mimicking advanced gallbladder carcinoma: A case report and review of literature. World J Gastroenterol 2006; 12: 2293-2296

9. Enomoto T, Todoroki T, Koike N, Kawamoto T, Matsumoto $\mathrm{H}$ : Xanthogranulomatous cholecystitis mimicking stage IV gallbladder cancer. Hepatogastroenterology 2003; 50: 1255-1258.

10. Koh T, Taniguchi H, Yamaguchi A, Kunishima S, Yamagishi H: Differential diagnosis of gallbladder cancer using positron emission tomography with fluorine-18-labeled fluoro-deoxyglucose (FDG-PET). J Surg Oncol 2003; 84: 74-81.

11. Rodriguez-Fernandez A, Gomez-Rio M, LlamasElvira JM, et al.: Positron-emission tomography with fluorine-18-fluoro-2-deoxy-D-glucose for gallbladder cancer diagnosis. Am J Surg 2004; 188: 171-175.

12. Anderson CD, Rice MH, Pinson CW, Chapman WC, Chari RS, Delbeke D: Fluorodeoxyglucose PET imaging in the evaluation of gallbladder carcinoma and cholangiocarcinoma. J Gastrointest Surg 2004; 8: 90-97.

13. Oe A, Kawabe J, Torii K, et al.: Distinguishing benign from malignant gallbladder wall thickening using FDG-PET. Ann Nucl Med 2006; 20: 699-703.

14. Petrowsky H, Wildbrett P, Husarik DB, et al.: Impact of integrated positron emission tomography and computed tomography on staging and management of gallbladder cancer and cholangiocarcinoma. J Hepatol 2006; 45: 43-50.

15. Makino I, Yamaguchi T, Sato N, Yasui T, Kita I: Xanthogranulomatous cholecystitis mimicking gallbladder carcinoma with a false-positive result on fluorodeoxyglucose PET. World J Gastroenterol 2009; 15: 3691-3693.

16. Xie XH, Xu HX, Xie XY, et al.: Differential diagnosis between benign and malignant gallbladder diseases with real-time contrast-enhanced ultrasound. Eur Radiol 2010; 20: 239-248.

17. McCoy JJ Jr, Vila R, Petrossian G, McCall RA, Reddy KS: Xanthogranulomatous cholecystitis. Report of two cases. J S C Med Assoc 1976; 72: 78-79.

18. Guzman-Valdivia G: Xanthogranulomatous cholecystitis in laparoscopic surgery. J Gastrointest Surg 2005; 9: 494-497.

19. Cardenas-Lailson LE, Torres-Gomez B, MedinaSanchez S, Mijares-Garcia JM, Hernandez-Calleros J: [Epidemiology of xanthogranulomatous cholecystitis]. Cir Cir 2005; 73: 19-23.

20. Yang T, Zhang BH, Zhang J, Zhang YJ, Jiang XQ, Wu MC: Surgical treatment of xanthogranulomatous cholecystitis: experience in 33 cases. Hepatobiliary Pancreat Dis Int 2007; 6: 504-508.

21. Goodman ZD, Ishak KG: Xanthogranulomatous cholecystitis. Am J Surg Pathol 1981; 5: 653-659.

22. Benbow EW: Xanthogranulomatous cholecystitis. Br J Surg 1990; 77: 255-256.

23. Srinivas GN, Sinha S, Ryley N, Houghton PW: Perfidious gallbladders-a diagnostic dilemma with xanthogranulomatous cholecystitis. Ann R Coll Surg Engl 2007; 89: 168-172.

24. Ramia JM, Muffak K, Fernandez A, Villar J, Garrote D, Ferron JA: Gallbladder tuberculosis: false-positive PET diagnosis of gallbladder cancer. World J Gastroenterol 2006; 12: 6559-6560.

25. Maldjian PD, Ghesani N, Ahmed S, Liu Y: Adenomyomatosis of the gallbladder: another cause for a "hot" gallbladder on 18F-FDG PET. AJR Am J Roentgenol 2007; 189: 36-38.

26. Meacock LM, Sellars ME, Sidhu PS: Evaluation of gallbladder and biliary duct disease using microbubble contrast-enhanced ultrasound. $\mathrm{Br} \mathrm{J}$ Radiol 2010; 83: 615-627.

27. Hirooka Y, Naitoh Y, Goto H, et al.: Contrastenhaceded endoscopic ultrasongography in gallbladder disease. Gastrointest Endosc 1998; 48: 406-410.

28. Numata K, Hiroyuki O, Morimoto $M$, et al: Differential diagnosis of gallbladder disease with contrast-enhanced harmonic gray scale ultrasonography. J Ultrasound Med 2007; 26: 763774 .

(Received, December 29, 2010)

(Accepted, February 17, 2011) 